\title{
Influence of deviations in the coil geometry on Wendelstein 7-X plasma equilibrium properties
}

\author{
T. Andreeva, S. Bozhenkov, V. Bykov, M. Endler, J. Fellinger, J. Geiger, M. Grahl, T. Klinger, \\ H. Thomsen \\ Max-Planck-Institut für Plasmaphysik, Teilinstitut Greifswald, Wendelsteinstraße 1, 17491 Greifswald, Germany
}

\begin{abstract}
Wendelstein 7-X (W7-X) is a modular advanced stellarator with a magnetic field optimized for good plasma confinement and stability, which went into operation in December 2015 at the Max-Planck-Institut für Plasmaphysik in Greifswald, Germany. The magnet system of W7-X consists of 70 superconducting coils with asbuilt-shapes and positions which deviate from the designed ones due to manufacturing and positioning tolerances as well as due to deformations under different kinds of loads. Step-by-step evaluation of the magnet system geometry during the machine construction included measurements of the winding pack geometry characterizing deviations of each coil filament caused by the fabrication process and the tracking of coil positions during main assembly steps. In addition finite element calculations were performed with help of the $360^{\circ}$ ANSYS Global Model (a finite element model, representing the whole W7-X magnet system, including all superconducting coils and their support structure) in order to define possible coil deformations under the dead-weight, cool-down and electromagnetic loads in different operation regimes. This paper shows the comparative analysis of different types of deviations in the coil geometry and discusses their influence on plasma equilibrium properties.
\end{abstract}

Keywords: Wendelstein 7-X, equilibrium, magnetic field, coil deformations, as-built coil geometry

\section{Introduction}

Wendelstein $7-\mathrm{X}$ is an advanced modular stellarator with nested magnetic surfaces created by a system of toroidally discrete coils which provide both toroidal and poloidal field components [1]. The magnet system of the machine consists of 50 non-planar (NPC) and 20 planar (PC) coils which are arranged in five identical modules (Fig. 1). In addition, each as-designed module obeys a stellarator (flip) symmetry of adjacent half-modules. In total, there are seven different types of coil geometries (five non-planar and two planar coils of one half module) to produce a large variety of magnetic configurations [2]. The superconducting coils are designed to produce a magnetic field strength of up to 3 $\mathrm{T}$ on the magnetic axis.

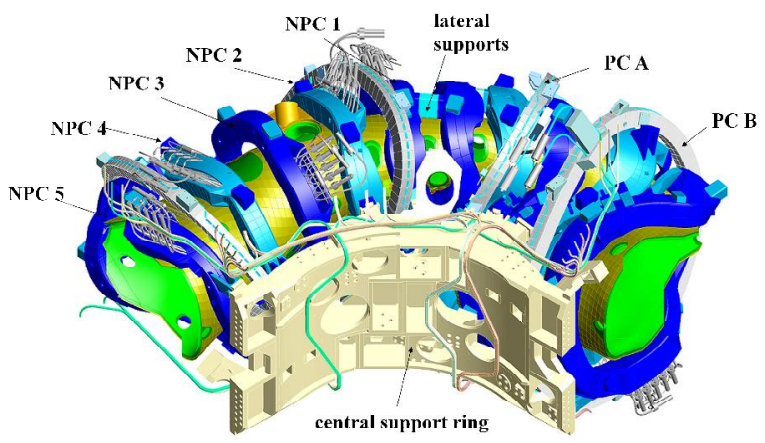

Fig. 1 One module of the W7-X coil system. PC: planar coil, type A or B; NPC: non-planar coil, type 1 to 5.

Preliminary simulations of the plasma equilibrium properties were performed taking into account asdesigned shapes and positions of W7-X coils. In these calculations each coil was represented by a single filament. As-built positions of coil filaments might considerably deviate from their as-designed values due to manufacturing and positioning tolerances or due to coil deformations under different kinds of loads.
Step-by-step evaluation of the magnet system geometry during the machine construction included measurements of the winding pack (WP) shape geometry in eight points characterizing deviations of each coil cross-section caused by the fabrication process as well as the tracking of coil positions during main assembly steps $[3,4]$. In addition finite element (FE) calculations were performed with help of the $360^{\circ}$ ANSYS Global Model [5], which allows to account for individual coil deformations, since the as-built geometry of W7-X magnet system do not obey 5 -fold symmetry. With help of this Model deformations of all 70 superconducting coils under the dead-weight, cool-down and electromagnetic loads in different operation regimes were simulated.

\section{Filament data collection}

The significant changes of the coil geometries are reflected in the following states of W7-X coordinate collection shown in Table. 1. Here each winding pack is represented by one filament, which consists of 96 points at the center of 96 WP cross-sections. Different variants of geometry states with the CAD abbreviation are based on the Computer-Aided Design (ideal) WP geometries. As-Built Geometry (ABG) states are WP geometries, obtained from measurements during the machine construction. FE1 are coil deformations calculated with finite element methods under bolt Preloads (PL), coil self weight (Dead Weight $=$ DW) and Cooling Down to $4 \mathrm{~K}$ temperatures (CD). FE2 abbreviation indicates the case with coil deformations under electromagnetic (EM) loads, which are specific for each operating case. In the first operating campaign of W7-X the special limiter magnetic configuration was investigated [6]. This OP1.1 configuration is named $\mathrm{J}$. It is characterized by equal winding currents in the NPCs and the PC winding 
currents being $39 \%$ of NPCs winding currents. For the FE-calculations of CAD_FE2 and ABG_FE2 geometries winding currents of $12.78 \mathrm{kA}$ for the NPCs and $4.98 \mathrm{kA}$ for the PCs were used, which correspond to the average magnetic field on axis of $2.5 \mathrm{~T}$.

Data set CAD_FE1 was obtained from the CAD_W geometries ("Warm" geometries at $20^{\circ}$ temperature) plus deformations due to $(\mathrm{PL}+\mathrm{DW}+\mathrm{CD})$, calculated in each WP cross-section by FE methods, and, in addition, plus shift $+6 \mathrm{~mm}$ in $\mathrm{z}$-direction in order to compensate the consequences of the $\mathrm{CD}$.

Data set CAD_FE2 was obtained from the CAD_W geometries plus deformations due to $(\mathrm{PL}+\mathrm{DW}+\mathrm{CD}+\mathrm{EM})$, calculated in each WP crosssections by FE methods, and, in addition, plus shift $+6 \mathrm{~mm}$ in $\mathrm{z}$-direction in order to compensate the consequences of the $\mathrm{CD}$.

Table 1. Collection of coil geometry states.

\begin{tabular}{|c|c|}
\hline $\begin{array}{l}\text { geometry state of } \\
\text { the coil system: } \\
\text { abbreviation /state } \\
\text { periodicity }\end{array}$ & description \\
\hline $\begin{array}{l}\text { CAD_W } \\
\text { 5-fold,flip }\end{array}$ & $\begin{array}{l}\text { Computer-Aided Design (CAD) } \\
\text { geometry at } 20^{\circ} \mathrm{C} \text { temperature }\end{array}$ \\
\hline $\begin{array}{l}\text { CAD_C } \\
\text { 5-fold, flip }\end{array}$ & CAD geometry at $4 \mathrm{~K}$ temperature \\
\hline $\begin{array}{l}\text { CAD_FE1 } \\
\text { 5-fold }\end{array}$ & $\begin{array}{l}\text { CAD_W geometries plus } \\
\text { deformations due to PL+DW+CD } \\
\text { CAD_W+PL+DW+CD }\end{array}$ \\
\hline $\begin{array}{l}\text { CAD_FE2 } \\
\text { 5-fold }\end{array}$ & $\begin{array}{l}\text { CAD_W geometries plus } \\
\text { deformations due to } \\
\text { PL+DW+CD+EM }(\mathrm{J} 2.5 \mathrm{~T})\end{array}$ \\
\hline $\begin{array}{l}\text { ABG_W } \\
\text { 1-fold }\end{array}$ & $\begin{array}{l}\text { As-Built Geometry measured at } \\
20^{\circ} \mathrm{C} \text { temperature (including real } \\
\text { PL+DW) }\end{array}$ \\
\hline $\begin{array}{l}\text { ABG_C } \\
\text { 1-fold }\end{array}$ & $\begin{array}{l}\text { As-Built Geometry at } 4 \mathrm{~K} \\
\text { temperature } \\
\text { CAD_C+(ABG_W-CAD_W) }\end{array}$ \\
\hline $\begin{array}{l}\text { ABG_FE1 } \\
\text { 1-fold }\end{array}$ & $\begin{array}{l}\text { As-Built geometries plus } \\
\text { deformations due to PL+DW+CD } \\
\text { CAD_FE1+(ABG_W- } \\
\text { CAD_W)-PL-DW }\end{array}$ \\
\hline $\begin{array}{l}\text { ABG_FE2 } \\
\text { 1-fold }\end{array}$ & $\begin{array}{l}\text { As-Built geometries plus } \\
\text { deformations due to } \\
\text { PL+DW+CD+EM (J } 2.5 \text { T) } \\
\text { CAD_FE2+(ABG_W - } \\
\text { CAD_W)-PL-DW }\end{array}$ \\
\hline
\end{tabular}

ABG_W data were obtained from the measurements, performed during WP-fabrication and assembly at the temperature $20^{\circ} \mathrm{C}$. At first, deviations in each WP crosssection due to WP fabrication tolerances were added to the CAD data. Then the best-fit transformation to the points measured during the assembly was applied. Since the measurements were performed on the already assembled coils, this data set contains deformations due to bolt preload and coil self weight.

ABG_C data were obtained from CAD_C geometries ("Cold" geometries at $4 \mathrm{~K}$ temperature) plus differences: ABG_W - CAD_W. Due to the measurements of the already assembled coils this data set contains deformations due to PL and DW.

ABG_FE1 data were obtained from CAD_FE1 geometries plus differences $\left(\mathrm{ABG}_{-} \mathrm{W}-\mathrm{CAD} \_\mathrm{W}\right)$ and minus rigid transformation from $\left(\mathrm{CAD}_{-} \mathrm{W}+\mathrm{PL}+\mathrm{DW}\right)$ state to CAD_W state in order to compensate for the shifts due to preload and deadweight, which are already contained in the As-built data.

ABG_FE2 data are obtained in the same procedure like for ABG_FE1, but now the starting point is CAD_FE2 instead of the previously used CAD_FE1.

Fig. 2 shows the differences in the position of the center of mass in z-direction for 7 coil types for the different geometry states. In the CAD_W and CAD_C geomteries the center of mass corresponds to the $\mathrm{z}=0$ plane for all coil groups. The largest offset of the center of mass is observed in the case of $\mathrm{ABG}_{-} \mathrm{C}$ and ABG_FE1 coordinates. Electromagnetic loads shift the coil system a bit in the upper direction and thus counteract the coil deformation due to their self weight.

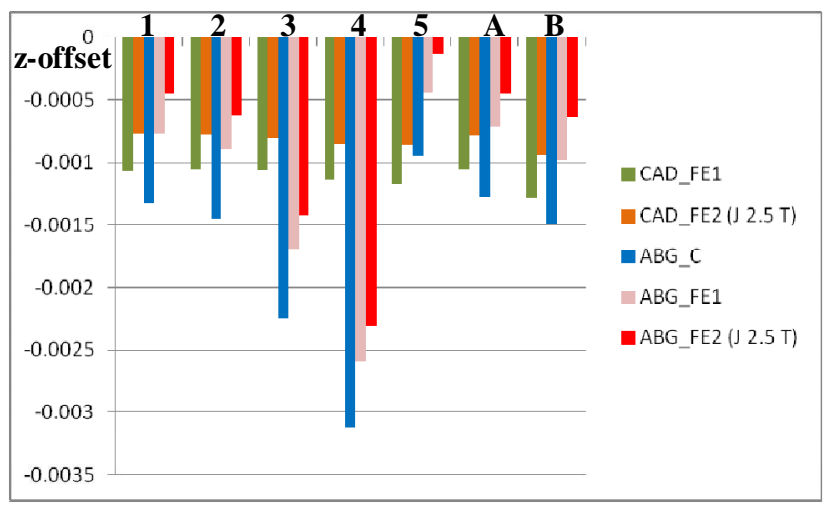

Fig. 2 (color online) Position of the center of mass in $\mathrm{z}-$ direction (z-offset, [m]) for each coil group in different geometry states with respect to CAD_W. 1-5 are NPC groups of types 1-5, 6- PCs of type A, 7- PCs of type B.

\section{Numerical approach}

The approach selected has included Biot-Savart fieldline-tracing calculations as well as VMEC [7] calculations for a stellarator-symmetric approximation to be prepared for finite beta studies. Since VMEC assumes the existence of nested flux surfaces, it is not possible to investigate the changes in the island topology, but other relevant equilibrium parameters as, for example, the rotational transform profile can be analyzed. The approach was to keep the five-fold and stellarator symmetry in VMEC-calculations on one hand and take into account individual deformations in the asymmetrical cases on the other hand. 
In order to remain as close as possible to the stellarator-symmetry, the offset described in section 2 was removed, separately for all WP filaments of PCs as one group and all WP filaments of NPCs as another group. Then, all $70 \mathrm{WP}$ filaments were rotated 5 times by a toroidal angle of $\varphi=72^{\circ}$ (always by one module) around the torus and then flipped. Thus, each WP was represented in the calculations by 10 filaments (700 filaments in total). This leads to a five-periodic stellarator-symmetric coil system. For calculations, each filament is represented by 97 poloidal cross-sections, where an additional cross-section at the end repeated the first one in order to close the current path.

These five-periodic, stellarator-symmetric coil geometries were used to generate the magnetic field data on a grid (so-called mgrid-files) for free-boundary VMEC calculations. In this way periodic and stellarator symmetric equilibrium calculations could be performed avoiding full-torus equilibrium calculations which are computationally very demanding.

\section{Modeling results}

Figures 3 and 4 show the results of the field line tracing for the CAD_C, ABG_C and ABG_FE2 (2.5 T J operating case) filament states. It is apparent, that the influence of coil deformation under electromagnetic loads in case of $2.5 \mathrm{~T}$ fields is significantly larger than that of as-built filament deviations alone.

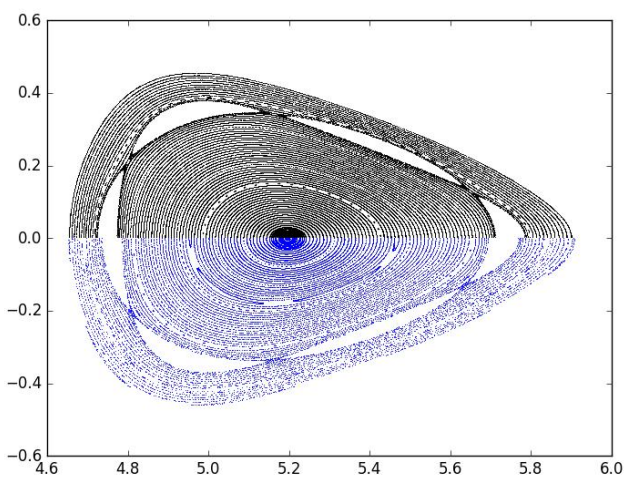

Fig. 3 (color online) Field line tracing for the $2.5 \mathrm{~T}$ J-operating case with CAD_C (black, upper half) and ABG_C (blue, lower half) filament geometries, which correspond to the ABG_C blue bars in Fig. 2.

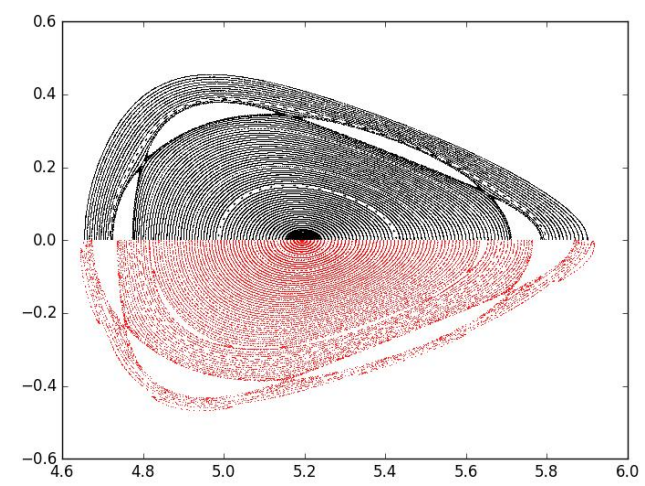

Fig. 4 (color online) Field line tracing for the $2.5 \mathrm{~T} \mathrm{~J}$-operating case with CAD_C (black, upper half) and ABG_FE2 (red, lower half) filament geometries, which correspond to the ABG_FE2 red bars in Fig. 2.

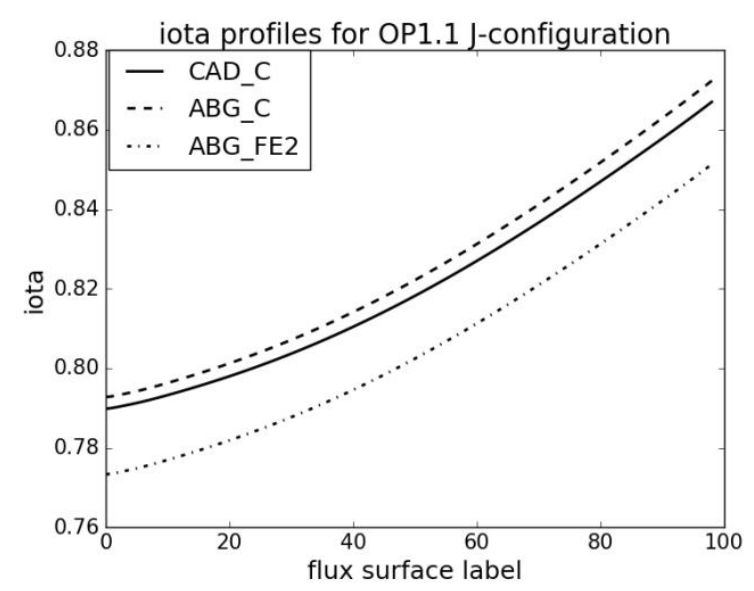

Fig. 5 Rotational transform profile for the $2.5 \mathrm{~T} \mathrm{~J}$ magnetic configuration. The solid black line corresponds to the CAD_C, dashed line - to ABG_C and dash-dotted line - to the ABG_FE2 filament geometries (the real situation during the experiment), that has the largest influence on the rotational transform profile.

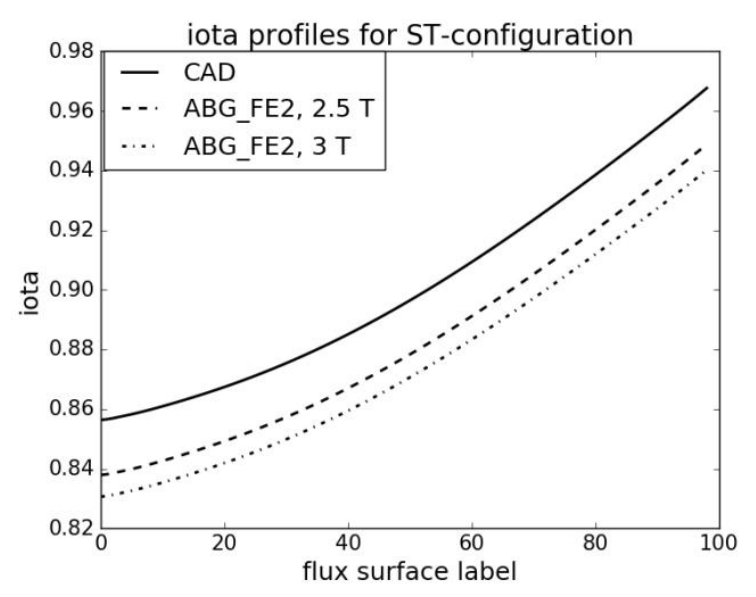

Fig. 6 Rotational transform profiles for the W7-X standard magnetic configuration with different filament geometries.

Changes in the rotational transform profile were analysed for the J-configuration at $2.5 \mathrm{~T}$ in case of CAD_C, ABG_C and ABG_FE2 coil geometries (Fig. $5)$. In the first $W 7-X$ operating campaign the plasma pressure was not very high, that is why these simulations were performed for the vacuum case, since the difference between small finite beta OP1.1 case and $\beta=0$ case was insignificant and would not influence the character of dependencies observed. One can see, that due to electromagnetic forces one can expect approximately $2 \%$ iota decrease in the operation cases with the magnetic field of $2.5 \mathrm{~T}$ and approximately $3 \%$ for the cases with $3 \mathrm{~T}$. This dependency was observed for the main magnetic configurations specified for W7-X and is illustrated in Fig. 6 for the standard operating case (foreseen in the next operational campaign) [2] with two different magnetic field strengths.

Fig. 7 shows the shift of the flux surfaces between VMEC calculations for $2.5 \mathrm{~T}$ J-configuration with CAD_C and ABG_FE2 coil shapes. The magnetic 
surfaces tend to become more circular under electromagnetic loads and the magnetic axis is shifted inwards in comparison with the simulation with CAD WP geometries. The same dependency was obtained also for other operating cases specified for W7-X. Table 2 represents the calculated shift of the plasma axis in the bean-shaped $\left(\varphi=0^{\circ}\right)$ and triangular $\left(\varphi=36^{\circ}\right)$ planes for the standard (ST), high mirror (HM) [2] and J 2.5 T cases.

Table 2. Shift of the plasma axis in $\varphi=0^{\circ}$ and $\varphi=36^{\circ}$ planes for $2.5 \mathrm{~T}$ standard (ST), high mirror (HM) and $\mathrm{J}$ operating cases.

\begin{tabular}{|l|l|l|l|}
\hline & ST case & HM case & J case \\
\hline $\begin{array}{l}\text { shift of plasma axis [mm], } \\
\varphi=0^{\circ} \text { plane }\end{array}$ & -6.3 & -5.5 & -4.5 \\
\hline $\begin{array}{l}\text { shift of plasma axis }[\mathrm{mm}], \\
\varphi=36^{\circ} \text { plane }\end{array}$ & -7.6 & -3.3 & -5.0 \\
\hline
\end{tabular}

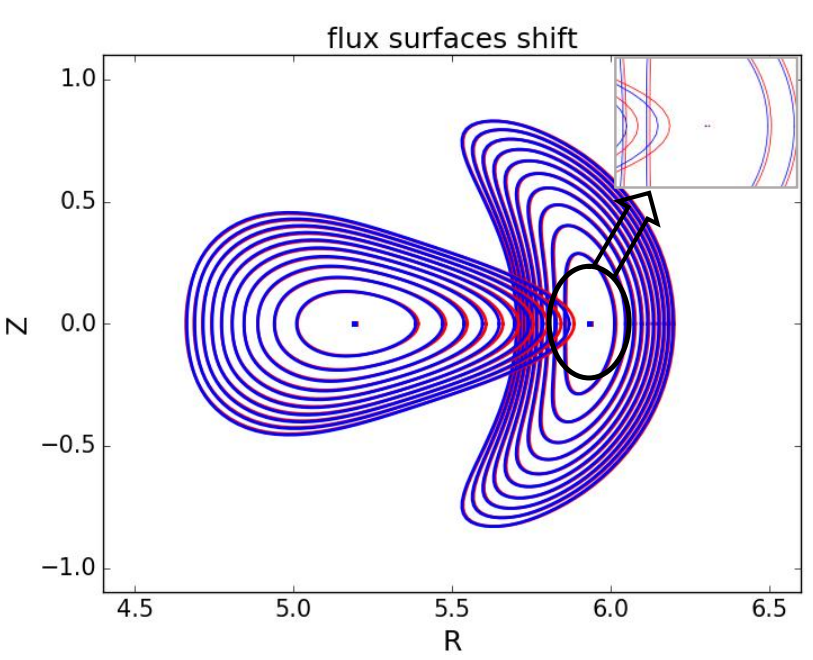

Fig. 7 Magnetic flux surfaces obtained from VMEC calculations with the CAD_C (red) and ABG_FE2 (blue) filament geometries for the J-operating case at $2.5 \mathrm{~T}$.

\section{Conclusions}

Sets of the main W7-X magnetic configuration geometries have been analyzed and collected in the data base, based on a single filament representation of the current path in the coils. These data have been made available via W7-X Webservices [8].

An approach to perform 5-fold symmetrical VMEC calculations on the basis of the as-built geometries of individual coils was developed. It allows to analyze the plasma equilibrium taking into account real coil shapes and positions avoiding at the same time time-consuming one field period VMEC studies.

It was found that iota values in case of calculations with as-built filament geometries deformed under electromagnetic loads decrease by approximately $2 \%$ in case of $2.5 \mathrm{~T}$ magnetic configurations and by $3 \%$ in case of $3 \mathrm{~T}$ configurations in comparison to the calculations with the as-designed filament shapes. These changes can be taken into account by the mapping of different diagnostics sensitive to the changes of the iota-profile and to the shift of the magnetic axis.

Next steps include further investigations of equilibrium properties, e.g. mirror ratio, in specified for W7-X magnetic configurations. Much more demanding task with respect to the computational resources will be a comparison of the studies presented with one-periodic VMEC calculations.

\section{Acknowledgments}

This work has been carried out within the framework of the EUROfusion Consortium and has received funding from the Euratom research and training programme 2014-2018 under grant agreement number 633053. The views and opinions expressed herein do not necessarily reflect those of the European Commission.

\section{References}

[1] G. Grieger, et al., "Modular stellarator reactors and plans for Wendelstein 7-X", Fusion Technol. 21 (1992) 17671778 .

[2] T. Andreeva, et al., "Characteristics of main configurations of Wendelstein 7-X", Problems of Atomic Science and Technology, Series: Plasma Physics, Vol. 4 (2002) 45-47.

[3] T. Brauer, et al., "Interaction of Metrology and Assembly at W7-X", IEEE Transactions on Plasma Science, Vol. 42, No. 7 (2014) 1943-1950.

[4] T. Andreeva, et al., "Tracking of the magnet system geometry during Wendelstein 7-X construction to achieve the designed magnetic field", Nuclear Fusion, Vol. 55, No. 6 (2015) 063025.

[5] V. Bykov, et al., "Specific Features of Wendelstein 7-X Structural Analyses", IEEE Transaction on Plasma Science, 42, No. 3 (2014) 690-697.

[6] T. S. Pedersen, et al., "Plans for the first plasma operation of Wendelstein 7-X", Nuclear Fusion, Vol. 55 (2015) 126001.

[7] S. Hirshman, W. van Rij, P. Merkel, "Three-dimensional free boundary calculations using a spectral green's function method", Comput. Phys. Comm., 43 (1986) 143-155.

[8] http://webservices.ipp-hgw.mpg.de/docs/services.html 